\title{
A practical alternative to calculating unmet need for family planning
}

This article was published in the following Dove Press journal:

Open Access Journal of Contraception

26 July 2017

Number of times this article has been viewed

\author{
Irit Sinai ${ }^{1,2}$ \\ Susan Igras' \\ Rebecka Lundgren' \\ 'Institute for Reproductive Health, \\ Georgetown University, Washington, \\ DC, USA; ${ }^{2}$ Palladium, Washington, DC, \\ USA
}

\begin{abstract}
The standard approach for measuring unmet need for family planning calculates actual, physiological unmet need and is useful for tracking changes at the population level. We propose to supplement it with an alternate approach that relies on individual perceptions and can improve program design and implementation. The proposed approach categorizes individuals by their perceived need for family planning: real met need (current users of a modern method), perceived met need (current users of a traditional method), real no need, perceived no need (those with a physiological need for family planning who perceive no need), and perceived unmet need (those who realize they have a need but do not use a method). We tested this approach using data from Mali $(n=425)$ and Benin $(n=1080)$. We found that traditional method use was significantly higher in Benin than in Mali, resulting in different perceptions of unmet need in the two countries. In Mali, perceived unmet need was much higher. In Benin, perceived unmet need was low because women believed (incorrectly) that they were protected from pregnancy. Perceived no need - women who believed that they could not become pregnant despite the fact that they were fecund and sexually active - was quite high in both countries. We posit that interventions that address perceptions of unmet need, in addition to physiological risk of pregnancy, will more likely be effective in changing behavior. The suggested approach for calculating unmet need supplements the standard calculations and is helpful for designing programs to better address women's and men's individual needs in diverse contexts.
\end{abstract}

Keywords: unmet need, family planning, contraception, Mali, Benin

\section{Introduction}

The number of women needing effective contraception worldwide rose from 716 million in 2003 to 867 million in $2012 .{ }^{1}$ Most of this increase (108 million) was due to population growth. The use of modern contraception also increased. ${ }^{1}$ However, while millions of women in the developing world are satisfied contraception users, an estimated 222 million have an unmet need for family planning. ${ }^{2}$ Despite the significant resources allocated to family planning in Central and West Africa over the past 20 years, unmet need remained essentially the same in these regions between 1990 and $2010 .^{3}$

One of the United Nations Sustainable Development Goals (SDG) is to improve health and well-being, in particular SDG 3 speaks of "preventing unintended pregnancy and reducing adolescent child bearing". ${ }^{4}$ Experts and global and national stakeholders worldwide agree that reducing unmet need for family planning is a priority and that meeting the need for contraception is one of the most cost-effective investments to alleviate poverty and improve health. ${ }^{5}$ But what, exactly, is unmet need?
Correspondence: Rebecka Lundgren Institute for Reproductive Health, Georgetown University, 1825

Connecticut Avenue NW, Suite 699,

Washington, DC 20009, USA

Tel +l 202687 I392

Email lundgrer@georgetown.edu 
The World Health Organization defines women with unmet need as "those who are fecund and sexually active but are not using any method of contraception, and report not wanting any more children or wanting to delay the birth of their next child". ${ }^{5}$ While the concept seems straightforward, operationalizing it into a measurable indicator to use in program design is complex, because calculations of need based solely on physiology may not correspond to individual understanding of need and their motivation to use or not use family planning. A complicating factor is the fact that the term "unmet need" usually applies to women only, because they are the ones who bear children. However, men are part of the equation and may also have unmet need for family planning.

Tékponon Jikuagou (TJ) is an initiative funded by the United States Agency for International Development to develop and test interventions to leverage social networks to address unmet need for family planning. The project began in 2010 in Mali, with formative research designed to understand what it is that prevents women and men who are sexually active and wish to avoid a pregnancy from using contraception. In the process, it became evident that the standard approach to calculating unmet need, while useful at the population level, is not adequately nuanced to design interventions to address unmet need for family planning because it does not sufficiently consider the individual's perception of his or her need. This paper proposes an alternate approach, developed to supplement the standard calculations of unmet need to address this gap. Following the 2012 political unrest in Mali, the project moved to Benin, where programs to address unmet need are being implemented and evaluated. This paper demonstrates the utility of the new approach using findings from both Mali and Benin. Before we do this, we present the two approaches.

In the description of the two approaches that follows (the standard approach and the suggested alternate approach), we use the terms "modern" and "traditional" family planning methods. The definition we use to distinguish the two is that used by the Demographic and Health Surveys (DHS). Modern methods include female and male sterilization, oral contraceptives, intrauterine devices, injectables, implants, male and female condoms, Standard Days Method, the Lactational Amenorrhea Method, and emergency contraception. Traditional methods include rhythm, withdrawal, and any other method mentioned spontaneously by the respondent (such as tinctures, potions, and herbs).

\section{The standard approach to calculating unmet need}

The standard approach to calculating unmet need was designed to view populations in the aggregate and answer the question, "if all demand for family planning were to be satisfied, how much might fertility be expected to decline?". ${ }^{6}$ The approach includes measurements of fecundity because these influence each woman's contribution to the total fertility rate.

The standard approach was revised by DHS in 2012 to simplify the calculations and allow for standardized unmetneed estimates using DHS (and other large surveys) globally. ${ }^{6}$ Since the publicly available DHS data for all countries already include unmet-need figures using the standard approach, it is easy for stakeholders to compare countries and regions and to assess progress over time. The current version uses fewer survey questions than the previous one and has achieved broad consensus among researchers, policy makers, program managers, and other stakeholders. ${ }^{7}$

Box 1 shows the questions included in the current version of the standard calculation of unmet need. More questions are often added to distinguish unmet need for spacing vs. limiting childbirth. Questions are shown here in the order they appear in the model DHS questionnaire, excluding skips and questions that are not relevant to calculating unmet need. Question numbers (in parentheses) correspond to the model DHS questionnaire Phase VI.

The standard calculation of unmet need categorizes women into four groups, using the questions depicted in Box $1 .{ }^{19}$

\section{Group I (met need)}

Women who were using a family planning method at the time of the survey. The definition distinguishes between need for spacing (women who wish to have another child in the future) and limiting (women who want to have no more children). While it can include women who are using a less effective traditional method, it usually refers only to women who use a modern method. We posit that women who are currently using a traditional method should be considered separately. They are not using a modern, effective method, yet they think that their need for family planning is met. Using the standard approach for calculating unmet need, they would be considered as having unmet need. But they themselves believe that their need is met. A recent study on the influence of natural method use on estimates of unmet need in Burkina Faso ${ }^{8}$ confirms that women who use traditional methods perceive that they are using family planning methods.

\section{Group 2 (unmet need)}

Women who are currently pregnant or in postpartum amenorrhea. If their current or recent pregnancy was unintended, they are considered to have unmet need. Only 
Box I Survey questions required to calculate unmet need for program design - standard approach

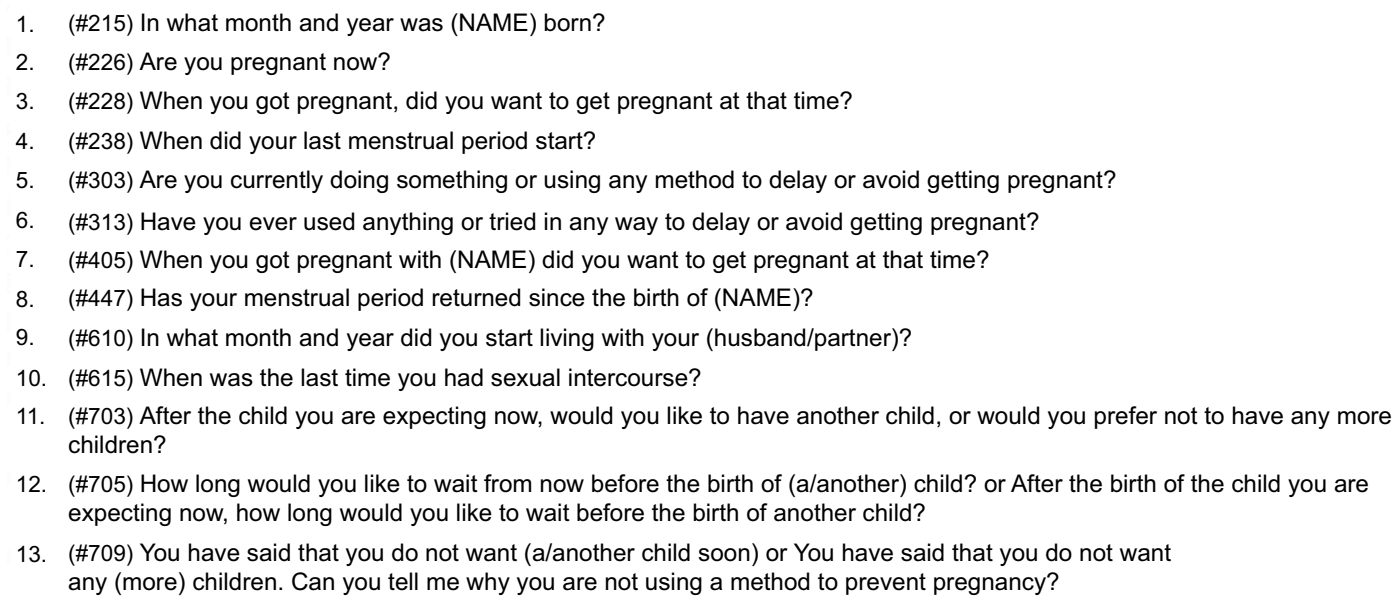

Notes: Survey questions reproduced from United States Agency for International Development (USAID). Demographic and Health Surveys Methodology. Questionnaires: household, woman's, and man's. [DHS Model Questionnaire - Phase 6 (2008-2013)]. Available from: http://dhsprogram.com/publications/publication-dhsq6-dhs-questionnairesand-manuals.cfm. Accessed July II, 2017. ${ }^{19}$ The numbers shown in parentheses are the original question numbers from the DHS questionnaire. To distinguish among women who wish to space or limit birth, add (\#229) Did you want to have a baby later or did you not want any (more) children?; (\#406) Did you want to have a baby later or did you not want any (more) children; and (\#704) Would you like to have (a/another) child or would you prefer not to have any (more) children?

Abbreviation: DHS, Demographic and Health Survey.

if their pregnancy was planned do they have no need. The reason for this is the expected downward bias on aggregate unmet need estimates of excluding these women, as their pregnancies were the result of prior unmet need. ${ }^{9}$ However, the solution of including pregnant women in the calculation is not ideal, because these retrospective reports of intendedness of pregnancies are likely to be downward biased, ${ }^{10}$ as retrospectively women often say that their current/recent pregnancy was intended, even if at the time of conception it was not. ${ }^{11}$ In our opinion, pregnant women do not always consider themselves as having unmet need, even if their pregnancy was unintended. While they could benefit from programs providing them information for future contraceptive use, they do not perceive that they currently have a need for family planning and will not take active steps to use a method during pregnancy. Another problem with this group is the duration of postpartum amenorrhea. In the standard calculation, women who are postpartum amenorrheic are assumed not to be at risk of pregnancy, and like pregnant women, their unmet need status is based on the wantedness of their most recent pregnancy. However, studies show that about a third of women can become pregnant before their first postpartum menses, in that they ovulate and their hormone levels can support pregnancy. ${ }^{12,13}$ Therefore, many women whose recent pregnancy was intended may actually have unmet need. On the other hand, many women in postpartum amenorrhea incorrectly perceive that they cannot become pregnant, especially if they are breastfeeding. These women do not think that they have a need for family planning and therefore do not take steps to obtain a method, despite their biological and physical need for a method.

\section{Group 3 (no need)}

Women who are infecund therefore have no need for a family planning method. These are women who are not pregnant, not in postpartum amenorrhea, and who either: 1) have been married 5 or more years, had no children in the past 5 years, and never used contraception; 2) respond "Can't get pregnant" on items regarding future desire for children; 3) said "menopausal/hysterectomy" as a reason for not using contraception; 4) responded to time since last period as $\geq 6$ month, and not postpartum amenorrheic; 5) responded to time since last period as "menopausal/hysterectomy" or "never menstruated", or "last period was before last birth", and last birth was over 5 years ago. ${ }^{14}$ Clearly, this group labels women as having no need for family planning if they have no real, biological need. But what about women who perceive that they have no need for family planning, while in fact they have a biological need? For example, a woman who thinks that she is infertile because she has had sexual intercourse infrequently for a period of time and has not become pregnant? Therefore, she will not seek a family planning method, even though she is fecund, is sexually active, and wishes to avoid pregnancy. The standard way of measuring unmet need does not distinguish these women. 


\section{Group 4 (unmet need)}

Women who are not pregnant or postpartum amenorrheic, not using a method, and are fecund (not in group 3). If they wish to become pregnant in the next 2 years, they are considered to have no need; if they do not wish to become pregnant, they have unmet need.

Clearly, while the standard calculations approach for unmet need is broadly used and accepted, it is not perfect, with issues related to the classification of pregnant women and women in postpartum amenorrhea, classification of traditional methods, and handling of sexual inactivity. ${ }^{7}$ Another concern is the lack of men's perspective. Estimates of unmet need for women and men do not always match, because some women are clandestine contraceptive users, and because husbands are typically more likely than wives to report the use of male methods..$^{15}$ The standard approach cannot be used for men because it is impractical to ask men infecundity questions.

\section{Suggested alternative for calculating unmet need for program design}

The TJ approach assumes that women's assessments of their own need for family planning is more likely than their physiological need to result in a decision to use contraception. This alternative is useful for policy-makers and program planners as they design programs to address women's need, although less useful than the standard approach to measure changes in overall fertility rates or to draw comparisons across regions and time. We developed this seven-question approach, which is intended to supplement the standard approach, through a formative research process, utilizing multiple methods including ethnography and structured interviews. It categorizes women into five current-need status groups, each with different programmatic needs: real met need, perceived met need, real no need, perceived no need, and perceived unmet need. The underlying assumption is that women's perceptions of their need are more likely to guide their behavioral choices than their physiological need.

- Real met need: women who are currently using a modern family planning method.

- Perceived met need: women who are currently using a traditional method. They think that their need is met, when in fact it is not because the "method" they use is less effective, or even completely ineffective. For example, women who use periodic abstinence, who believe that they should avoid unprotected sex to prevent pregnancy in the days immediately following their period (and feel they are "safe" precisely when they are more likely to conceive), and those who believe that they are protected from pregnancy if they drink salt water after having sex or insert spider webs into their vagina.

- Real no need: women who are currently pregnant or desire a child in the next year, women who are not sexually active, and women who had a hysterectomy or are postmenopausal.

- Perceived no need: women who think that they are not fecund, despite being physiologically fecund. This includes, for example, women who have sex infrequently, and women with children older than 6 months who believe that they cannot become pregnant because they are in postpartum amenorrhea.

- Perceived unmet need: women who do not fall into any of the other categories. They realize that they are at risk of pregnancy, wish to not become pregnant, and yet are not using a method, modern or traditional. They may have no access to services or to the method of their choice, or they may not feel empowered to use a method.

Figure 1 shows a graphic representation of the suggested approach. Box 2 shows the survey questions required to assign women a current need status per this approach. This approach can also be used with men, with one important difference. In societies where polygamy is prevalent, men can be assigned more than one current need status, as they may have a different need status with each wife. In societies where polygamy is not practiced, the measures for women and men can be directly compared.

\section{Materials and methods}

To demonstrate the suggested approach, we show data from two studies. The first was part of the formative research in Mali. The complete adult populations of two villages were listed, and all women of reproductive age were interviewed in both villages $(n=425)$. The study was undertaken in a village in Bandiagara Health District (Mopti region) and another in Koutiala Health District (Sikasso region). We do not provide the names of the villages to maintain confidentiality of respondents, as all adults in the villages participated in the study.

The second study is from the baseline survey in Benin, which will eventually be compared to an endline survey to evaluate the interventions of the $\mathrm{TJ}$ initiative. A representative sample of 1080 women of reproductive age were interviewed in the Couffo region. All participants provided written informed consent. Data collection instruments for both stud- 


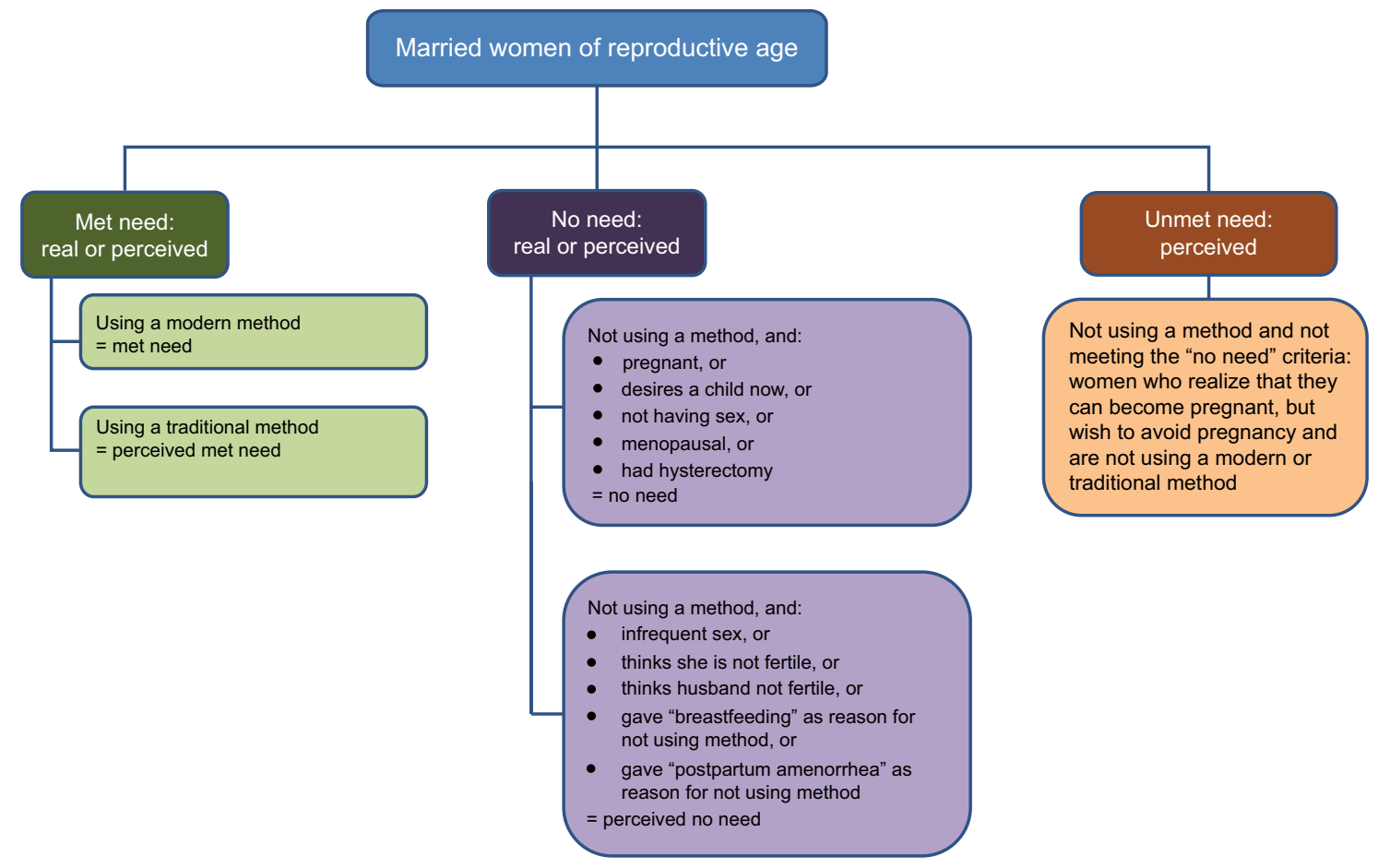

Figure I Tékponon Jikuagou's approach for calculating unmet need for program design.

Box 2 Survey questions required to calculate unmet need for program design: Tékponon Jikuagou approach.

1. Are you pregnant now? (yes = real no need)

2. (if no to \# 1) Would you like to become pregnant within the next 12 months? (yes = real no need)

3. (if no to \#2) Are you currently doing something to delay or avoid getting pregnant? (yes = met need, real or perceived)

4. (if yes to \#3) Which method are you using? (modern = real met need; traditional = perceived met need)

5. (if no to \#3) Is it possible for you to become pregnant?

6. (if no to \#5) Why do you say that? (real or perceived no need, depending on reason)

7. (if yes to \#5) You said that you do not want to become pregnant this year, but you are not using any method to avoid pregnancy. Please tell me why (perceived unmet need or perceived no need, depending on reason)

ies were similar, and included, among others, the questions required to calculate need status for the suggested approach. All protocols and instruments for both studies were approved by the Georgetown University Institutional Review Board, the Comité National d'Ethique pour la Santé et les Sciences de la Vie in Mali, and the Comité d'Ethique de la Recherche de l'Institut des Sciences Biomédicales Appliquées in Benin.

\section{Results}

Table 1 shows the distribution of need status, per the suggested approach, in Mali and Benin. The design of this alternate approach resulted from our program design and evaluation needs and was not the purpose of this research. Therefore, our instruments did not include all the questions needed to calculate unmet need per the standard approach (ie,
Table I Need status

\begin{tabular}{lll}
\hline Need status (\%) & Mali $(\mathbf{n}=\mathbf{4 2 5})$ & Benin $(\mathbf{n}=1080)$ \\
\hline Real no need & 33.9 & 32.8 \\
Perceived no need & 19.9 & 23.6 \\
Real met need & 10.4 & 13.9 \\
Perceived met need & 0.9 & 18.6 \\
Perceived unmet need & 34.8 & 11.1 \\
\hline
\end{tabular}

last time she had sex and day of the last menstrual period), and we cannot directly compare figures based on the two approaches. The 2006 DHS survey in Mali's Mopti and Sikasso regions (regions of the two villages) shows an unmet need of $22.5 \%$ and $29.5 \%$ of married women, respectively; the 2011-2012 DHS survey in Benin shows $26.0 \%$ of unmet need in the Couffo region. ${ }^{16}$ 
Very little modern method use (real met need) was observed, and the percentage of women with real or perceived no need in both countries was quite similar. However, significant differences emerged in traditional method use (perceived met need). There was much traditional method use in Benin but very little in Mali. Perceived unmet need was significantly higher in Mali than in Benin.

\section{Discussion}

\section{Findings and interpretation}

We presented an alternate approach to calculating unmet need, which is most useful for the design of behavior change interventions. We showed how using this approach results in very specific program implications for Mali and Benin that would not have become evident using the standard approach.

It is not surprising that perceived unmet need in Mali (34.8\%) was higher than the published data for the two regions of Mali $(22.5 \%$ and $29.5 \%$ ), because the study focused on hard-to-reach rural villages in the country, where unmet need is especially high. At first glance, the perceived unmet need rate for Benin appears low (only 11.1\%). However, if we add to it the $18.6 \%$ of women who were using ineffective traditional methods (perceived met need), we get a figure that is quite similar to the unmet need reported in the DHS for the region (26.0\%). Traditional method users would be considered to have unmet need per the standard approach.

\section{Strengths and weaknesses of the approach}

The suggested approach for calculating unmet need was not designed to replace the current standard calculation; it serves a different purpose. The standard calculation is useful for comparisons between countries and regions, and examining progress at the population level over time, while the alternate approach suggested here is useful for program design and helps identify the types of programs that would most benefit women and men in project communities. The proposed approach can be calculated in relatively quick community-based surveys that include only seven survey questions, none of them very personal (women do not need to respond to questions about their sexual activity or menstrual period), compared to the longer, more complex and intrusive questionnaire needed for the traditional approach.

Another advantage of the proposed approach is that it can be applied as easily to men as to women. Recent studies show the importance of calculating unmet need for men, as well as women, ${ }^{17,18}$ and understanding the nuanced differences between them.

\section{Implications of the findings}

This new approach was developed to move from a description of unmet need to program-relevant estimates that can help with program design. While ultimately total estimates may be comparable for the two approaches, the new approach presented here provides information to help develop programs to meet the specific nature of unmet need in their catchment areas. Our findings, therefore, have programmatic implications. Given the high level of traditional method use in Benin, couples in this country would benefit from programs that build on current spacing practices, addressing concerns related to modern methods, comparing efficacy of modern to traditional methods, improving access to quality services, and expanding method choice to include options that appeal to traditional method users.

About $20 \%$ of women in both countries had perceived no need. For the most part, these are women who had an actual need for family planning (they wished to avoid pregnancy, were sexually active, yet were not using a method), but they thought they could not become pregnant. Examples include women who believed that they could not become pregnant because they only had sex infrequently, and women with children older than 6 months, who thought that they could not become pregnant because they were still breastfeeding or in postpartum amenorrhea. These women would benefit from programs that teach couples about their fertility. For example, information could include the days in the cycle in which women can become pregnant, the fact that men are fertile all the time, that infrequent sexual activity can still result in pregnancy, and that breastfeeding women can conceive. This would enable couples to correctly assess the probability of pregnancy across the reproductive life course, including the postpartum period, post abortion, and while breastfeeding, encouraging the use of family planning when it is most needed.

Finally, the $34.8 \%$ of women in Mali and $11.1 \%$ of women in Benin who had perceived unmet need per this calculation approach would benefit from two general types of programs. Women in Mali and Benin who perceived that they had an unmet need for family planning but were unwilling to use available methods would benefit from an expanded method mix. Those with perceived unmet need who did not use family planning because of their husband's opposition or because of stigma associated with family planning use could benefit from community-level interventions that address gender norms including male hegemony and reproduction as the primary way to express masculinity and femininity. 


\section{Conclusion}

The alternative approach presented here is not intended to replace the standard approach for calculating the rates of unmet need, but to supplement it, specifically for program design and implementation. Future research may compare the two approaches directly - through the administration of a survey questionnaire that includes both sets of questions. This exercise will be most useful if collected before and after the implementation of a program based on results of calculations per the new approach.

\section{Acknowledgments}

This work was supported by the United States Agency for International Development (USAID) under cooperative agreement: No. AID-OAA-A-10_00066. The views expressed by the authors do not necessarily reflect the views or policies of USAID or of Georgetown University.

\section{Disclosure}

Irit Sinai is currently employed by Palladium. The authors report no conflicts of interest in this work.

\section{References}

1. Darroch JE, Singh S. Trends in contraceptive need and use in developing countries in 2003, 2008, and 2012: an analysis of national surveys. Lancet. 2012;381(9879):1756-1762.

2. Population Reference Bureau (PRB). World Population Data Sheet 2012. Washington, DC: PRB; c2015. Available from: http://www.prb. org/Publications/Datasheets/2012/world-population-data-sheet/factsheet-unmet-need.aspx. Accessed July 11, 2017.

3. Alkema L, Kantorova V, Menozzi C, Biddlecom A. National, regional, and global rates and trends in contraceptive prevalence and unmet need for family planning between 1990 and 2015: a systematic and comprehensive analysis. Lancet. 2013;381(9878):1604-1606.

4. United Nations (UN). Sustainable Development Goal 3. Ensure healthy lives and promote well-being for all at all ages. Available from: http:// sustainabledevelopment.un.org.sdg3. Accessed July 21, 2017.
5. World Health Organization (WHO). Unmet need for family planning [cited December 9, 2013]. Available from: http://www.who.int/reproductivehealth/topics/family_planning/unmet_need_fp/en/. Accessed July 11, 2017.

6. Bradley EK, Croft TN, Fishel JD, et al. Revising unmet need for family planning. DHS analytical studies No. 25. Calverton, Maryland: ICF International; 2012.

7. Cleland J, Harbison S, Shah IH. Unmet need for contraception: issues and challenges. Stud Fam Plann. 2014;45(2):105-122.

8. Rossier C, Senderowicz L, Soura A. Do natural methods count? Underreporting of natural contraception in urban Burkina Faso. Stud Fam Plann. 2014;45(2):171-182.

9. Westoff CF, Ochoa L. Unmet need and the demand for family planning. Unmet need and the demand for family planning. DHS Comparative Studies No. 5. Calverton, MD: Macro International; 1991.

10. Casterline JB, Sinding SW. Unmet need for family planning in developing countries and implications for population policy. Popul Dev Rev. 2007;26(4):691-723.

11. Koenig MA, Acharya R, Singh S, Roy TK. Do current measurement approaches underestimate levels of unwanted childbearing? Evidence from rural India. Pop Stud (Camb). 2006;60(3);243-256.

12. Campbell OM, Gray RH. Characteristics and determinants of postpartum ovarian function in women in the United States. Am J of Obstet Gynecol. 1993;169(1):55-60.

13. Arévalo M, Jennings V, Sinai I. Application of simple fertility awarenessbased methods of family planning to breastfeeding women. Fertil Steril. 2003;80(5):1241-1248.

14. Bradley SE, Casterline JB. Understanding unmet need: history, theory, and measurement. Stud Fam Plann. 2014;45(2):123-150.

15. Becker S, Costenbader E. Husbands' and wives' reports of contraceptive use. Stud Fam Plann. 2005;32(2):111-129.

16. ICF International, 2012. The DHS Program STATcompiler [cited March 28, 2012]. Available from: www.statcompiler.com.

17. Pearson E, Becker S. Couples' unmet need for family planning in three West African countries. Stud Fam Plann. 2014;45(3): 339-359.

18. Rosenberg J. Men's data are needed to estimate unmet need among couples. Int Perspect Sex R H. 2014;40(40):217-218.

19. United States Agency for International Development (USAID). Demographic and Health Surveys Methodology. Questionnaires: household, woman's, and man's. [DHS Model Questionnaire - Phase 6 (2008-2013)]. Available from: http://dhsprogram.com/publications/ publication-dhsq6-dhs-questionnaires-and-manuals.cfm. Accessed July $11,2017$.
Open Access Journal of Contraception

\section{Publish your work in this journal}

Open Access Journal of Contraception is an international, peerreviewed, open access, online journal, publishing original research, reports, reviews and commentaries on all areas of contraception. In addition to clinical research, demographics and health-related aspects, the journal welcomes new findings in animal and preclinical studies

\section{Dovepress}

relating to understanding the biological mechanisms and practical development of new contraceptive agents. The manuscript management system is completely online and includes a very quick and fair peer-review system. Visit http://www.dovepress.com/testimonials.php to read real quotes from published authors. 